\title{
The prevalence and distribution of the atherosclerotic plaques in the abdominal aorta and its branches
}

\author{
C. Günenç Beşer ${ }^{1}$, M. Karçaaltıncaba², H.H. Çelik ${ }^{1}$, R. Başar ${ }^{1}$ \\ ${ }^{1}$ Department of Anatomy, Faculty of Medicine, Hacettepe University, Ankara, Turkey \\ 2Department of Radiology, Faculty of Medicine, Hacettepe University, Ankara, Turkey
}

[Received: 13 November 2016; Accepted: 14 December 2015]

\begin{abstract}
Background: The aim of our study was to determine the existence, distribution, type and burden of the atherosclerotic plaques which are found in the abdominal aorta, its branches and the branching points.

Materials and methods: Two hundred and sixty-one patients (117 female, 144 male; mean age 53.34 \pm 16.02 , range 12-84) who underwent dual-source computed tomography angiography (CTA) were retrospectively analysed. The prevalence and distribution of the plaques in the proximal, middle and distal parts of abdominal aorta and its branches; coeliac trunk, superior and inferior mesenteric arteries, renal arteries, splenic artery and common, external and internal iliac arteries and in the aortic orifices, the type and severity of these plaques and their relations with age and gender were studied. Results: In our study, 69.3\% of the patients had atherosclerotic plaques, mostly at the distal part of abdominal aorta. The existence of the plaques increased with age. The types of these plaques were mixed (43\%), calcified (24\%) and soft (3\%). Mixed and calcified plaques were more common in the abdominal aorta and its branches, respectively. All of the arteries except for inferior mesenteric artery mostly had mild plaques. The plaques at the branching points, which were most frequently localised in the aortic bifurcation, were found in the $41.8 \%$ of the patients. The plaques in the branches were usually accompanied by atherosclerosis of abdominal aorta.

Conclusions: Dual-source CTA enables mapping of atherosclerotic burden in abdominal arteries. Knowing the localisation, type and severity of the atherosclerotic plaques can be important to predict the clinical results and choose the proper treatment. (Folia Morphol 2016; 75, 3: 364-375)
\end{abstract}

Key words: abdominal aorta, atherosclerosis, atherosclerotic plaques, dual-source computed tomography angiography, vascular anatomy

\section{INTRODUCTION}

Atherosclerosis remains the major cause of mortality and morbidity in developed countries. Atherosclerosis typically occurs over many years. Growth of atherosclerotic plaques develops discontinuously, with silent periods intervened by periods of rapid evolution. In general, after a prolonged silent period, it may manifest clinically. Clinical manifestations of atherosclerosis may be chronic, as stable or effortinduced angina pectoris, intermittent claudication, or acute, as myocardial infarction, stroke or sudden cardiac death. Many people may show no clinical expressions of atherosclerosis in lifetime [20].

Atherosclerotic plaques occur in particular areas in the vasculature and clinical manifestations may vary according to the affected vessel. Atherosclerotic

Address for correspondence: Dr C. Günenç Beşer, MD, PhD, Department of Anatomy, Faculty of Medicine, Hacettepe University, Sihhiye, 06100, Ankara, Turkey, tel: +90-312-3052117, fax: +90-312-3107169, e-mail: crngnc5er@gmail.com 
plaques of the coronary arteries are mostly found at the proximal part of anterior interventricular artery (left anterior descending). Proximal part of renal arteries, bifurcation of common carotid arteries may also be affected. Atherosclerotic lesions are mostly seen at abdominal aorta and the branching points where the blood stream breaks down. Although it is widely known that the abdominal aorta and its branching points are the most common places for atherosclerotic lesions, recent studies were mostly focused on the coronary arteries.

The purpose of our study was to determine the presence and distribution of the atherosclerotic plaques in the abdominal aorta, its branches, common, internal and external iliac arteries and the branching points. We examined 64 multi-sliced computed tomography (CT) of 261 patients (117 female; 144 male). Our study was achieved retrospectively and with this study we aimed to provide a detailed anatomical and clinical study to lead the further examinations about this subject.

\section{MATERIALS AND METHODS}

\section{Patient population}

Two hundred and sixty-one patients ( 117 females and 144 males, mean age $53.34 \pm 16.02$, range 12-84) who underwent dual-source computed tomography angiography (DS-CTA) for any reason at our hospital were retrospectively analysed. In our study, the only parameters of the patients that were evaluated were age and gender. No additional radiation dose was applied to the patients. Ethics committee approval was received by Hacettepe University Medical Faculty Ethics Committee, Faculty of Health Sciences (Number: HEK 08/158-41). Analyses were performed within the routine clinical standards of our institution. This retrospective study evaluated existing clinical data and records. No additional procedures were performed.

\section{DS-CTA in the diagnosis of atherosclerosis}

Dual-source computed tomography angiography offers significant advantages over more conventional imaging methods in the evaluation of the mesenteric vasculature. With DS-CTA, the collimation is thinner and scanning is faster, this prevents artefacts of motion and breathing. And also DS-CTA is cheaper, minimum invasive and with lower radiation exposure. This method provides excellent anatomical visualisation of the main mesenteric trunks and the smaller vessels.
Additionally, the composition and the severity of the atherosclerotic plaques can be effectively differentiated with this method $[16,17]$. Siemens Definition $2 \times 64$ sliced dual-source computed tomography (Erlangen, Germany) was used; 120 cc, 300 mg/dL of ionic contrast material was injected through an automatic injector to the patients. Slice thickness was $1 \mathrm{~mm}$. Axial images were transferred to a separate workstation (Leonardo; Siemens, Germany) with advanced volume-rendering software. The DS-CTA data were reviewed on a workstation with multi-planar reformatting (MPR), maximum intensity projections (MIP) and volume rendering technique (VRT).

\section{The parameters}

In this study, age and gender of the patients, presence of the atherosclerotic plaques in the particular arteries or branching points and also the type and the size of the plaques were evaluated.

Patient age ranged from 12 to 82 years, and they were classified into eight age groups: 10-20 years, 21-30 years, $31-40$ years, $41-50$ years, $51-60$ years, 61-70 years, 71-80 years, and over 81 years.

The prevalence and the distribution of the atherosclerotic plaques in the abdominal aorta and its branches were studied. The abdominal aorta was subdivided into three parts: the proximal part was between $12^{\text {th }}$ thoracic and $2^{\text {nd }}$ lumbar vertebrae, the middle part was between the $2^{\text {nd }}$ and $3^{\text {rd }}$ lumbar vertebrae and the distal part was between the $3^{\text {rd }}$ and $4^{\text {th }}$ lumbar vertebrae. The branches of the abdominal aorta that were included in the study were coeliac trunk $(\mathrm{CT})$, superior mesenteric artery (SMA), right and left renal arteries (RRA, LRA), and inferior mesenteric artery (IMA). The continuation of abdominal aorta, the common iliac arteries $(\mathrm{CIA})$ and its branches, external (EIA) and internal iliac arteries (IIA) were also examined. The splenic artery (SA), a branch of the $\mathrm{CT}$, was also evaluated. Renal arteries, splenic artery, common, external and internal iliac arteries were also subdivided into proximal $1 / 3$, middle $1 / 3$ and distal $1 / 3$ parts. The presence of the atherosclerotic plaques on the branching points of these arteries (the ostia of the CT, SMA, IMA, RRA, LRA, EIA and IIA, and aortic bifurcation) were also studied.

The types of these plaques were classified as calcified, soft or mixed plaques. In the DS-CTA, calcified atherosclerotic plaques were hyperdense and soft plaques were hypodense. The mixed plaques included the calcified plaques as well as the soft plaques. 
Additionally, the severities of these plaques were noted. Atherosclerosis was rated by relative intimal area of involvement. The sizes of the plaques were divided into three groups, taking the size of the vessels into consideration. The plaques in the abdominal aorta were classified as mild $(0-1 \mathrm{~cm})$, medium $(1-2 \mathrm{~cm})$ and severe $(>2 \mathrm{~cm})$. For the plaques in the branches of the abdominal aorta; mild plaques were between $0 \mathrm{~cm}$ and $0.5 \mathrm{~cm}$, the medium ones were between $0.5 \mathrm{~cm}$ and $1 \mathrm{~cm}$ and the severe plaques were larger than $1 \mathrm{~cm}$.

\section{Statistical analysis}

First of all, the frequency tables and the crosstabs were evaluated. Pearson's $\chi^{2}$ test was used to verify the results. Fisher's exact test was used when the parametric hypothesis had not been provided. McNemar and Cochran's Q tests were performed for determining the relations between the vessels. The significance level of 0.05 was used $(p<0.05)$. Bonferroni correction was used for the comparisons between the vessels. Analyses were performed by using SPSS 11.

\section{RESULTS}

\section{The prevalence and distribution of the}

\section{atherosclerotic plaques}

The atherosclerotic plaques were found in 132 of 261 patients $(50.6 \%)$ in the distal part, in 112 (42.9\%) patients in the middle part and in 90 (34.5\%) patients in the proximal part of abdominal aorta. The differences between the proximal part and the other parts were statistically significant $(p=0.018)$. Eight of $261(3.1 \%)$ patients had plaques in the CT, $9(3.4 \%)$ of them had plaques in the SMA and $7(2.7 \%)$ of them had plaques in the IMA.

One hundred and twelve (42.9\%) patients had plaques in the right common iliac artery (RCIA) and 101 (38.7\%) patients had plaques in the left common iliac artery (LCIA). Sixty-nine (26.4\%) patients had plaques in the right external iliac artery (REIA) and more plaques were found in the distal part of this artery $(p<0.001)$ : $23.8 \%$ in the distal, $11.5 \%$ in the middle and $10.3 \%$ in the proximal part. The plaques in the right internal iliac artery (RIIA) were found in 79 (30.3\%) patients: $25.3 \%$ proximal, $19.5 \%$ middle, $15.3 \%$ distal. The plaques were found in $58(22.2 \%)$ patients in the left external iliac artery (LEIA): $9.6 \%$ proximal, $10 \%$ middle, $19.5 \%$ distal. More plaques were found in the distal part, which was statistically significant. Eighty-six (33\%) patients had plaques in the left internal iliac artery (LIIA): $26.1 \%$ proximal, $21.8 \%$ middle, $18.8 \%$ distal. There was significant difference between proximal and distal parts. More plaques were found in the LIIA than the LEIA. Also, more plaques were found in CIAs than EIA and IIA $(p<0.001)$.

Twenty-three of $261(14.6 \%)$ patients had atherosclerotic plaques in the RRA: $6.5 \%$ proximal, $2.7 \%$ middle, $2.3 \%$ distal and $26(10 \%)$ of them had plaques in the LRA: $7.7 \%$ proximal, $1.5 \%$ middle, $1.9 \%$ distal. Both arteries' proximal parts had more plaques than the other parts $(p<0.001)$.

The atherosclerotic plaques of the SA were detected in $38(14.6 \%)$ patients: $5.4 \%$ proximal, $10.3 \%$ middle, $11.9 \%$ distal. Less plaques were found in the proximal part $(p<0.001)$.

The percentages of the atherosclerotic plaques in the abdominal aorta and its branches independent from age and gender are shown in Figure 1.

\section{The presence of the atherosclerotic plaques with respect to age groups}

The presence of the atherosclerotic plaques with respect to age groups is shown in Table 1. According to this table, the percentages of the presence of the atherosclerotic plaques increased with age (Figs. 2, 3). There was a statistical significance between the age groups in respect to atherosclerotic plaque presence $(p<0.001)$.

\section{The presence of the atherosclerotic plaques with respect to gender}

In the study, 75 of 117 (64.1\%) females and 106 of $144(73.6 \%)$ males had atherosclerotic plaques. There was no statistically significant difference between males and females $(p=0.52)$.

The difference between males and females with respect to plaque presence was statistically significant in distal part of abdominal aorta and throughout RCIA, LCIA, REIA, LEIA, RIIA and LIIA. In these branches more plaques were found in males.

In 41-50, 51-60, 61-70 age groups there was a statistically significant difference between males and females, in favour of males, for the presence of the plaques in RCIA, LCIA, REIA and RIIA. In LEIA, there was a significant difference between genders in 51-60, 61-70, 71-80 age groups and in LIIA, the significant difference was in the 51-60, 61-70 and over 81 age groups. Both arteries had more atherosclerotic plaques in males. 


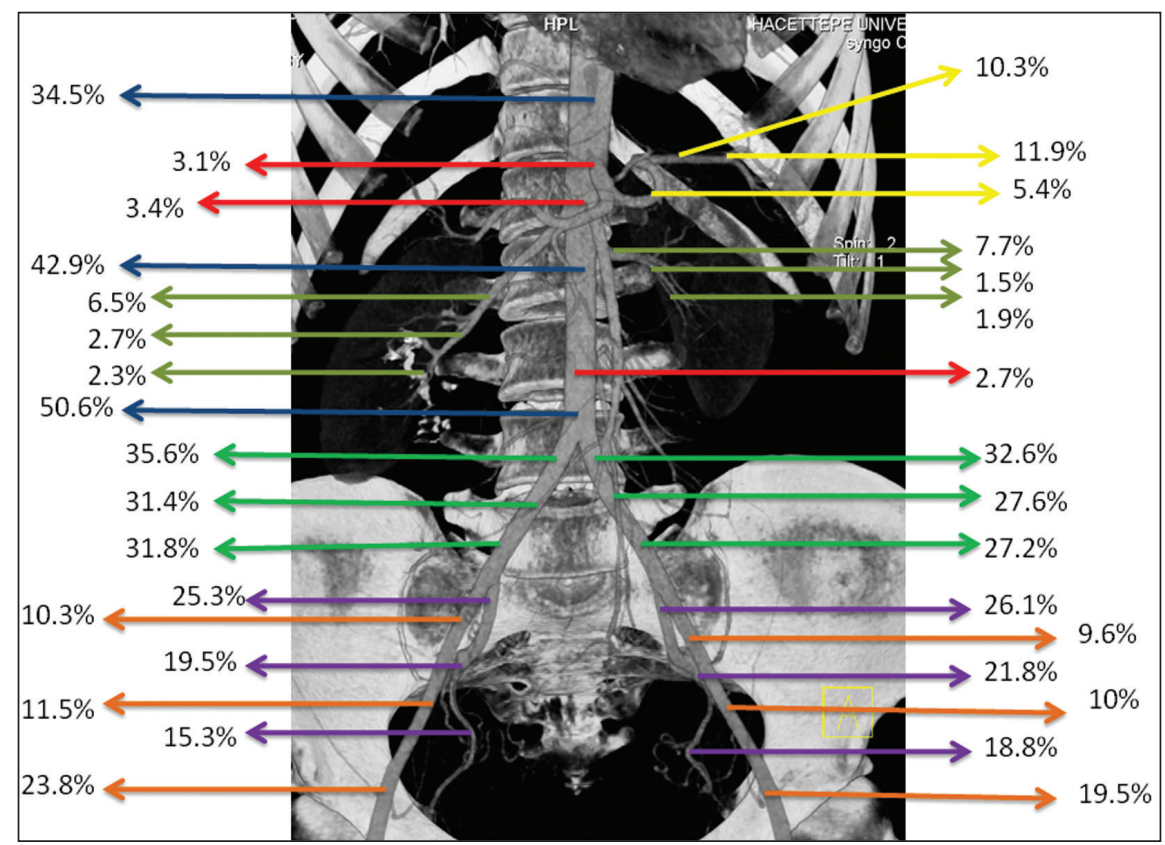

Figure 1. The prevalence and distribution of the atherosclerotic plaques in abdominal aorta and its branches. On the left (top-down): Proximal part of abdominal aorta; coeliac trunk; superior mesenteric artery; middle part of abdominal aorta; proximal, middle and distal parts of right renal artery; distal part of abdominal aorta; proximal, middle and distal parts of right common iliac artery; proximal, middle and distal parts of right internal and external iliac arteries; On the right (top-down): Middle, distal and proximal parts of splenic artery; proximal, middle and distal parts of left renal artery; inferior mesenteric artery; proximal, middle and distal parts of left common iliac artery; proximal, middle and distal parts of left internal and external iliac arteries.

Table 1. The presence of the atherosclerotic plaques with respect to age groups

\begin{tabular}{|c|c|c|c|}
\hline \multirow[t]{2}{*}{ Age groups } & \multicolumn{2}{|c|}{ Plaques } & \multirow[t]{2}{*}{ Total (\%) } \\
\hline & None (\%) & Present (\%) & \\
\hline $0-20$ & $13 / 100 \%$ & - & $13 / 100 \%$ \\
\hline $21-30$ & $11 / 84.6 \%$ & 2/15.4\% & $13 / 100 \%$ \\
\hline $31-40$ & $22 / 81.5 \%$ & $5 / 18.5 \%$ & $27 / 100 \%$ \\
\hline $41-50$ & $19 / 40.4 \%$ & 28/59.6\% & $47 / 100 \%$ \\
\hline $51-60$ & $14 / 19.2 \%$ & $59 / 80.8 \%$ & $73 / 100 \%$ \\
\hline $61-70$ & $1 / 1.9 \%$ & $52 / 98.1 \%$ & $53 / 100 \%$ \\
\hline $71-80$ & - & $30 / 100 \%$ & $30 / 100 \%$ \\
\hline$\geq 81$ & - & $5 / 100 \%$ & $5 / 100 \%$ \\
\hline Total & $80 / 30.7 \%$ & 181/69.3\% & $261 / 100 \%$ \\
\hline
\end{tabular}

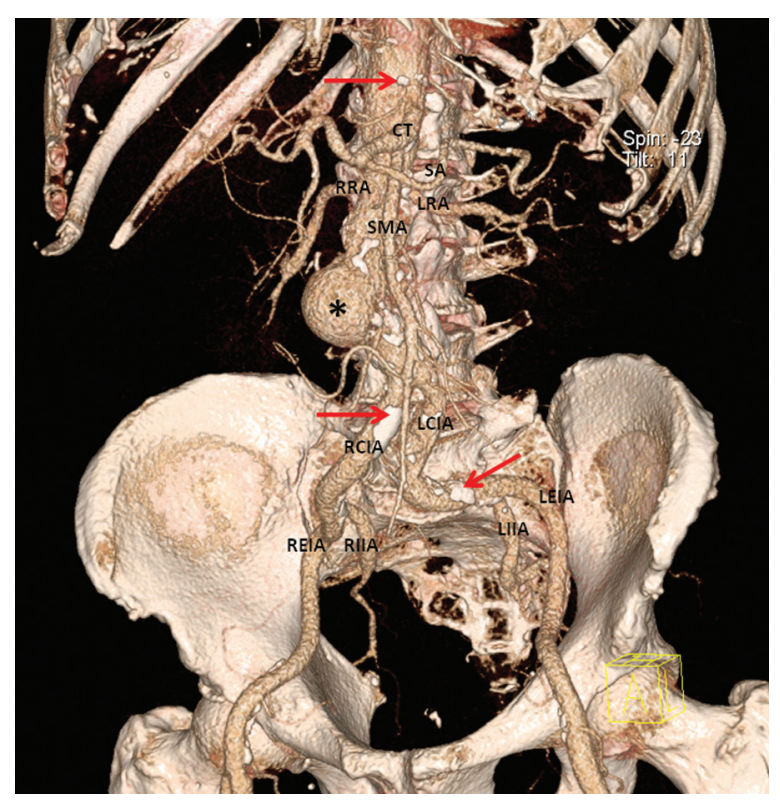

Figure 2. 82-year-old male, dual-source computed tomography angiography volume rendered; atherosclerotic plaques were indicated with arrows; CT — coeliac trunk; SA — splenic artery; RRA — right renal artery; LRA — left renal artery; SMA — superior mesenteric artery; LCIA — left common iliac artery; RCIA — right common iliac artery; LEIA — left external iliac artery; LIIA — left internal iliac artery; REIA — right external iliac artery; RIIA — right internal iliac artery; *aneurysm. 


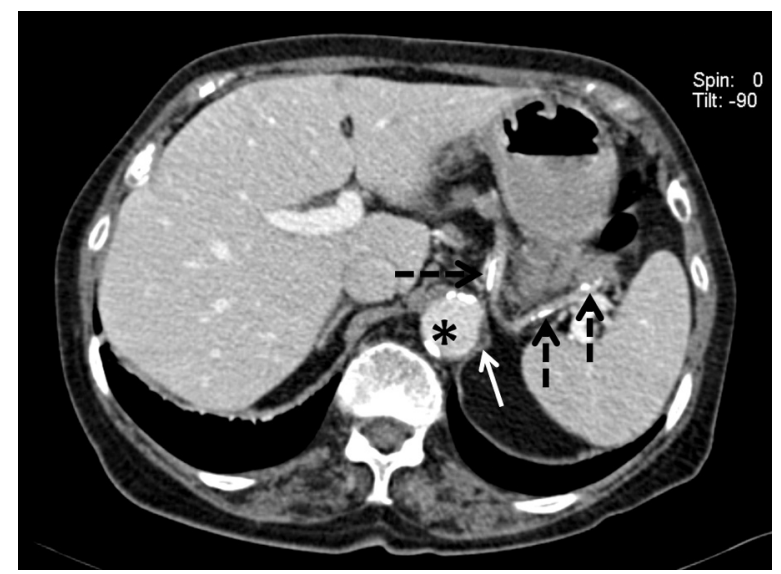

Figure 3. Atherosclerotic plaques with respect to age groups; 75-year-old female, dual-source computed tomography angiography multi-planar reformatting; black dash arrows - calcified atherosclerotic plaques in the proximal, middle and distal parts of splenic artery; white arrow - soft atherosclerotic plaque in the proximal part of abdominal aorta; ${ }^{*}$ calcified atherosclerotic plaque in the proximal part of abdominal aorta.

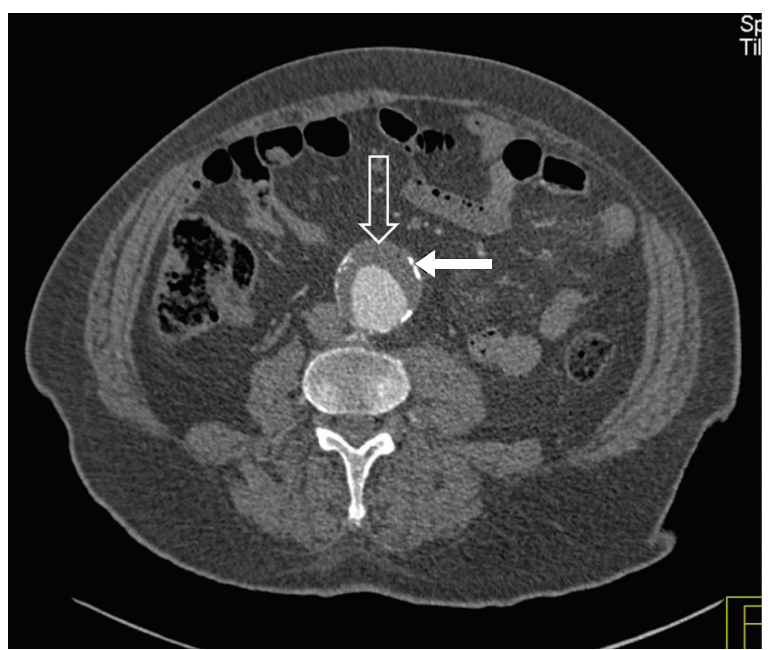

Figure 4. The type of the atherosclerotic plaques; 71-year-old male, dual-source computed tomography angiography multi-planar reformatting; white open arrow - severe, soft atherosclerotic plaques in the distal part of abdominal aorta; white arrow - mild, calcified atherosclerotic plaques in the distal part of abdominal aorta.

Table 2. The types of the atherosclerotic plaques for the branches and parts of the abdominal aorta

\begin{tabular}{lcccc}
\hline The vessel & Calcified & Soft & Mixed & Total \\
\hline Aorta, proximal/\% & $26 / 28.9 \%$ & $19 / 21.1 \%$ & $45 / 50 \%$ & $90 / 100 \%$ \\
Aorta, middle/\% & $31 / 27.7 \%$ & $10 / 8.9 \%$ & $71 / 63.4 \%$ & $112 / 100 \%$ \\
Aorta, distal/\% & $43 / 32.6 \%$ & $5 / 3.8 \%$ & $84 / 63.6 \%$ & $132 / 100 \%$ \\
CT/\% & $8 / 100 \%$ & - & - & $8 / 100 \%$ \\
SMA/\% & $9 / 100 \%$ & - & - & $9 / 100 \%$ \\
IMA/\% & $7 / 100 \%$ & - & - & $7 / 100 \%$ \\
SA/\% & $38 / 100 \%$ & - & $38 / 100 \%$ \\
RRA/\% & $22 / 95.7 \%$ & $1 / 4.3 \%$ & $23 / 100 \%$ \\
LRA/\% & $26 / 100 \%$ & - & $26 / 100 \%$ \\
RCIA/\% & - & $35 / 29.9 \%$ & $117 / 100 \%$ \\
LCIA $\%$ & $78 / 66.7 \%$ & $33 / 32.7 \%$ & $101 / 100 \%$ \\
REIA/\% & $62 / 61.4 \%$ & $15 / 21.7 \%$ & $69 / 100 \%$ \\
LEIA/\% & $51 / 73.9 \%$ & $6 / 5.9 \%$ & $14 / 24.1 \%$ & $58 / 100 \%$ \\
RIIA/\% & $40 / 69 \%$ & $3 / 4.3 \%$ & $17 / 21.5 \%$ & $79 / 100 \%$ \\
LIIA/\% & $60 / 75.9 \%$ & $4 / 6.9 \%$ & $18 / 20.9 \%$ & $86 / 100 \%$ \\
\hline
\end{tabular}

CT — coeliac trunk; SMA — superior mesenteric artery; IMA — inferior mesenteric artery; SA — splenic artery; RRA — right renal artery; LRA — left renal artery; RCIA — right common iliac artery; LCIA — left common iliac artery; REIA — right external iliac artery; LEIA — left external iliac artery; RIIA — right internal iliac artery; LIIA — left internal iliac artery

\section{The type of the atherosclerotic plaques}

In our study, there were 181 (69.3\%) patients with atherosclerotic plaques; 62 (23.75\%) of them had only calcified, $7(2.68 \%)$ of them had only soft and 112 (42.9\%) of them had mixed plaques (Fig. 4). According to these findings, most common type was mixed type and the least common type was soft type and these results were statistically significant $(p<0.001)$.

The plaque types for each vessel were shown in Table 2. According to the results, most commonly mixed plaques were found throughout the abdominal aorta. However, in the CT, SMA, IMA, SA, RRA, LRA, RCIA, 
LCIA, REIA, LEIA, RIIA and LIIA calcified plaques were the most common type of atherosclerotic plaques.

\section{The burden of the atherosclerotic plaques}

Throughout the abdominal aorta and in all of the branches that were included in the study, except for the IMA, mild plaques were the most common type. Second common type was medium plaques and the least common type was severe plaques for these arteries except for the distal part of abdominal aorta, SMA and IMA (Fig. 5). For the distal part of abdominal

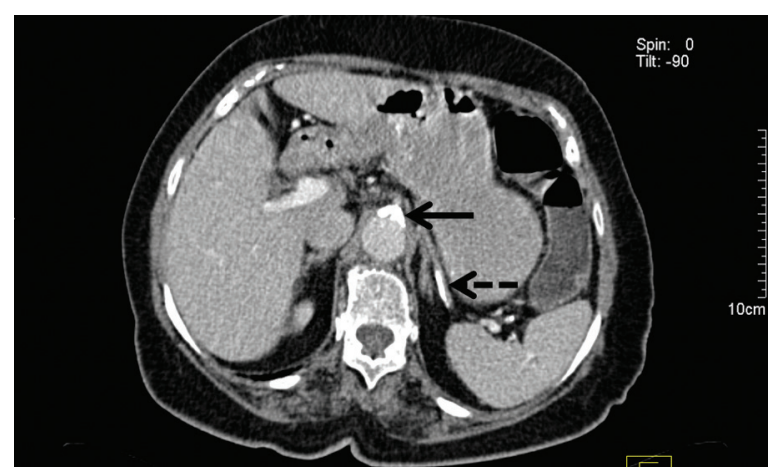

Figure 5. The burden of the atherosclerotic plaques; 80-year-old female, dual-source computed tomography angiography multiplanar reformatting; black arrow - severe, calcified plaque in the proximal part of abdominal aorta and in the aortic ostium of coeliac trunk; black dash arrow - severe, calcified plaques in the middle part of splenic artery. aorta, the second common type was severe and the least common was medium plaques ( $p<0.001$ ). The results for SMA and IMA were not statistically significant. The prevalence of the severity of atherosclerotic plaques is shown in Table 3.

The burden of the atherosclerotic plaques did not increase with age.

\section{The presence of the atherosclerotic plaques in the} branching points

One hundred and nine of 261 (41.8\%) patients had atherosclerotic plaques in the branching points. No plaques were found in 0-30 age group. The prevalence of the plaques were $0.4 \%$ in $31-40$ age group, $2.3 \%$ in $41-50$ age group, $11.5 \%$ in $51-60$ age group, $15.3 \%$ in $61-70$ age group, $10.7 \%$ in $71-80$ age group and $1.5 \%$ in patients over 81 years (Fig. 6). Plaque presence in the branching points increased with age. The percentage decreased over 81 because of insufficient number of patients in that age group.

Sixty-three of 144 male (43.8\%) and 46 of 117 (39.3\%) female patients had plaques in the branching points. There was not any statistically significant difference between genders.

Eighty (80) of 109 patients (73.4\%) had plaques in the aortic bifurcation. The plaques in the ostia of $\mathrm{CT}$, EIAs, IIAs, SMA, RAs and IMA were found in $45.9 \%$

Table 3. The severity of the atherosclerotic plaques

\begin{tabular}{lcccc}
\hline The vessel & Mild plaques & Medium plaques & Severe plaques & Total \\
\hline Aorta, proximal/\% & $57 / 63.3 \%$ & $28 / 31.1 \%$ & $5 / 5.6 \%$ & $90 / 100 \%$ \\
Aorta, middle/\% $\%$ & $27 / 24.1 \%$ & $16 / 14.3 \%$ & $112 / 100 \%$ \\
Aorta, dista/\% $\%$ & $31 / 23.5 \%$ & $39 / 29.5 \%$ & $132 / 100 \%$ \\
CT/\% & $69 / 61.6 \%$ & $1 / 12.5 \%$ & - & $8 / 100 \%$ \\
SMA/\% & $62 / 47 \%$ & - & $2 / 22.2 \%$ & $9 / 100 \%$ \\
IMA/\% & $7 / 87.5 \%$ & $4 / 57.1 \%$ & - & $7 / 100 \%$ \\
SA/\% & $7 / 77.8 \%$ & $9 / 23.7 \%$ & $5 / 13.2 \%$ & $38 / 100 \%$ \\
RRA/\% & $3 / 42.9 \%$ & $7 / 30.4 \%$ & $3 / 13 \%$ & $23 / 100 \%$ \\
LRA/\% & $24 / 63.2 \%$ & $9 / 34.6 \%$ & - & $26 / 100 \%$ \\
RCIA/\% & $13 / 56.5 \%$ & $37 / 31.6 \%$ & $24 / 20.5 \%$ & $117 / 100 \%$ \\
LCIA/\% $\%$ & $31 / 30.7 \%$ & $22 / 21.8 \%$ & $101 / 100 \%$ \\
REIA/\% & $17 / 65.4 \%$ & $9 / 13 \%$ & $69 / 100 \%$ \\
LEIA/\% & $56 / 47.9 \%$ & $6 / 10.3 \%$ & $58 / 100 \%$ \\
RIIA/\% & $48 / 47.5 \%$ & $7 / 8.9 \%$ & $79 / 100 \%$ \\
LIIA/\% & $43 / 62.3 \%$ & $20 / 34.5 \%$ & $96 / 100 \%$ \\
\hline
\end{tabular}

CT — coeliac trunk; SMA — superior mesenteric artery; IMA — inferior mesenteric artery; SA — splenic artery; RRA — right renal artery; LRA — left renal artery; RCIA — right common iliac artery; LCIA — left common iliac artery; REIA — right external iliac artery; LEIA — left external iliac artery; RIIA — right internal iliac artery; LIIA — left internal iliac artery 


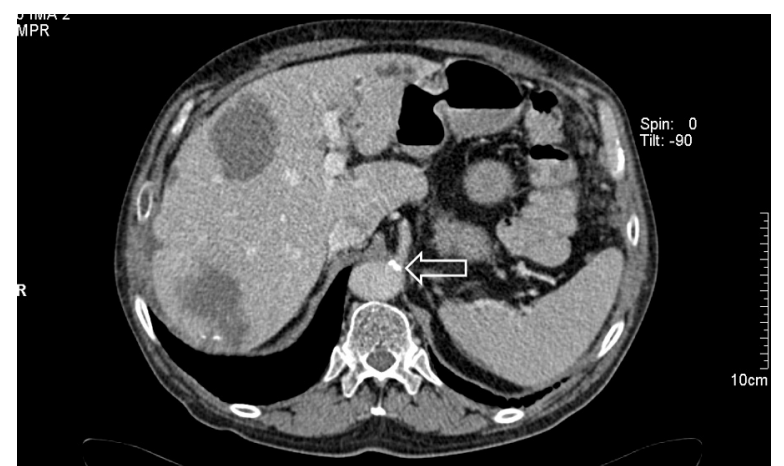

Figure 6. The atherosclerotic plaques in the branching points; 60 -year-old male, dual-source computed tomography angiography multi-planar reformatting; white open arrow — severe, calcified plaque in the aortic ostium of coeliac trunk.

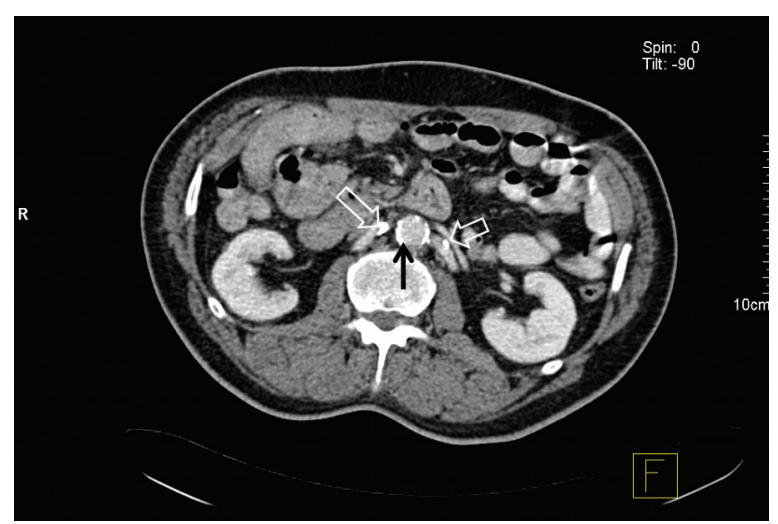

Figure 7. The co-occurrence of the atherosclerotic plaques in the middle part of abdominal aorta and the renal arteries; black arrow — calcified atherosclerotic plaques in the middle part of abdominal aorta; white open arrow - calcified plaques in the proximal parts of renal arteries.

(50 patients), 26.6\% (29 patients), 25.7\% (28 patients), 22\% (24 patients) and 8.3\% (9 patients), respectively. The plaques were most commonly found at the aortic bifurcation; second common localisations were the ostia of CT and the least common localisations were the ostia of IMA ( $p<0.001)$.

The co-occurrence of the atherosclerotic plaques in the abdominal aorta and the main branches

In our study, the co-occurrence of the atherosclerotic plaques in the proximal, middle, distal parts of abdominal aorta and CT, SMA, IMA, RRA, LRA and SA were also evaluated.

When the patients with plaques in the CT, SMA and IMA were examined, it was determined that $62.5 \%, 100 \%$ and $57.1 \%$ of them had also plaques in the proximal, $75 \%, 88.9 \%$ and $42.9 \%$ of them had plaques in the middle and $87.5 \%, 100 \%$ and $71.4 \%$ of them had plaques in the distal parts of abdomi- nal aorta, respectively. For patients with plaques in the RRA and LRA, $87 \%$ and $80.3 \%$ of them had also plaques in the proximal, $78.3 \%$ and $69.2 \%$ of them had plaques in the middle and $87 \%$ and $92.3 \%$ of them had plaques in the distal parts of abdominal aorta, respectively. For the patients with plaques in the SA, $81.6 \%$ of them had plaques both in the proximal and middle parts and $89.5 \%$ of them had plaques in the distal part of abdominal aorta (Fig. 7).

\section{DISCUSSION}

The cerebral, coronary and renal circulations have been widely studied by surgeons, whereas the mesenteric circulation was the least remarkable part of the circulatory system that supplies the organs essential for life. Although, most of the digestive system does not consist of essential organs, the intestinal circulation takes larger amount of the cardiac output and also the stenosis of the main arteries in the intestinal circulation may cause a more certain and quick death [22].

Atherosclerosis is a systemic inflammatory disease which characteristically starts from the branching points of the arteries. Haemodynamic factors play a part in the localisation of the atherosclerosis $[6,7$, 11]. Abdominal aorta is the site for earliest and most severe atherosclerotic disease. The extent of the lesions in aorta may reflect the propensity of a patient to form atheroma [12].

DeBakey et al. $[6,7]$ classified the atherosclerotic occlusive disease according to the distribution of the lesions into five major categories: (I) the coronary arteries, (II) the major branches of the aortic arch, (III) the visceral branches of abdominal aorta (CT, SMA, renal arteries), (IV) the terminal abdominal aorta and its major branches, (V) a combination of two or more of these categories. The localisation of atherosclerosis also determines the prognosis of the stenosis and the treatment options $[6,7,11]$.

\section{The prevalence and distribution of the} atherosclerotic plaques

Allison et al. [2] reported that $73 \%$ of 6814 patients had abdominal aortic calcium in their multiethnic study. According to DeBakey et al. [6, 7], atherosclerotic occlusive disease of the terminal abdominal aorta (infrarenal portion) and iliac arteries (category IV) was the most common, approximately two-fifths of the series. Occlusive disease of the visceral branches of abdominal aorta (category III) was the least fre- 
quent one (3\%). For category III, renal arteries were the most common atherosclerotic branches; lesions were mainly found in the dorsal part of abdominal aorta and in the proximal inflow tracts. They reported that atherosclerotic occlusive disease in all categories generally developed in the proximal or mid-proximal parts of the arterial bed $[6,7,11]$. Many other authors also reported that especially the lower two-thirds of abdominal aorta (infrarenal, distal part) is prone to both aneurysmal and occlusive atherosclerosis and also this part contains the most severe lesions in both sexes and in all decades [15, 24, 25, 31]. In our study, $69.73 \%$ (182 patients) of 261 patients had atherosclerotic plaques; $50.6 \%$ of these plaques were in the distal, $42.9 \%$ were in the middle and $34.5 \%$ were in the proximal part of abdominal aorta. According to these results, plaques were more commonly found in the distal part of abdominal aorta and these results were compatible with the literature.

Atherosclerotic plaques tend to occur at the ostia of the vessels and in the first $2-3 \mathrm{~cm}$ of the main trunks $[9,8,18]$. Reiner et al. noted that the plaques tend to be in the first 9-12 cm of the SMA (cited in Dick et al., 1967 [9]). The different point in Maljatzkaja's study was the splenic artery which had the plaques throughout its length (cited in Dick et al., 1967 [9]). SA, CT, mesenteric and renal arteries had less plaques than any other vessels $[25,24]$. Derrick et al. [8] examined CT and SMA and found that 44\% of the CT and $37 \%$ of the SMA showed narrowing secondary to atherosclerosis just distal to the aortic orifice. Jarvinen et al. [18] reported that 67\% (80 patients) of the patients had aortic atherosclerosis, $29 \%$ (35 patients) of them had stenosis in the CT, SMA or IMA. Hansen et al. [13] evaluated the mesenteric arteries in patients older than 65 years by visceral duplex sonography and found that $17.5 \%$ of them had mesenteric artery stenosis, $15 \%$ of them had CT stenosis, $1.3 \%$ had CT and SMA stenosis together and $0.9 \%$ had SMA stenosis. In our study, 8 of 261 patients $(3.1 \%)$ had plaques in the CT, 9 (3.4\%) of them had plaques in the SMA and 7 (2.7\%) of them had plaques in the IMA. Also less plaques were found in the proximal part of SA in comparison with the other parts.

Renal artery stenosis sites were commonly located in the proximal parts (74\%) and the ostia of the arteries [12]. Aggarwal et al. [1] found the main site for atherosclerotic lesions in renal arteries were near the ostia (85\%) and the second site was the proximal part of the arteries (27\%), middle part was the least involved part. RRA had atherosclerotic lesions in $77 \%$, whereas LRA had lesions in $88 \%$. In our study, $14.6 \%$ of the patients had atherosclerotic plaques in the RRA and $10 \%$ of them had plaques in the LRA. Both arteries had more plaques in their proximal parts (RRA; $6.5 \%$ and LRA; $7.7 \%)$; these results were statistically significant and compatible with the literature.

\section{Atherosclerotic plaques with respect to age groups}

The prevalence and extent of fatty streaks and fibrous plaques increase rapidly with increasing age $[11,21]$. The prevalence and volume of the plaques increased with age in both sexes [23-25, 29]. In the CORD study, calcification scores increased rapidly with age [15]. DeBakey et al. [6, 7] reported that aging was a risk factor for atherosclerotic occlusive disease in category I, II and IV. Jarvinen et al. [18] found that aortic atherosclerosis was strongly associated with aging; $67 \%$ of the patients aged 81 or more had mesenteric artery stenosis, whereas $6 \%$ aged less than 40 years old had mesenteric artery stenosis. According to the study of Aggarwal et al. [1], atherosclerotic plaques were found in $75 \%$ of renal arteries in the fourth decade, in $85 \%$ in the fifth decade, in $70 \%$ in the sixth decade and in $100 \%$ in the seventh decade. Renal artery calcium was also significantly associated with age [3]. In the literature, some of the studies claimed that the plaques were not correlated with age [13].

In our study, no plaques were found in the 0-20 age group, whereas $15.4 \%$ of the patients in $21-30$ age group, $18.5 \%$ of the patients in $31-40$ age group, $59.6 \%$ of the patients in $41-50$ age group, $80.8 \%$ of the patients in 51-60 age group, $98.1 \%$ of the patients in 61-70 age group and all of the patients over 81 years old had atherosclerotic plaques. There was a statistical significance between the age groups $(p<0.001)$ and the plaque presence increases with respect to age which is compatible with most of the recent studies.

\section{Atherosclerotic plaques with respect to gender}

DeBakey et al. [6, 7] found that males predominated in all of the categories. According to the Framingham Heart Study [23], in non-hypertensive group the prevalence of the plaques was greater in women, 
whereas in hypertensive group no significant difference was determined between genders. The prevalence of calcium for all vessels was lower in women than men. The prevalence of the calcified plaques for men and women were $66 \%$ and $35 \%$ in iliac arteries and $60 \%$ and $48 \%$ in abdominal aorta, respectively [10]. According to Roberts et al. [24, 25], there was a significant difference between males and females in the distribution and severity of atherosclerosis only in the fourth decade and only in coronary, iliac and renal arteries. Aggarwall et al. [1] reported that $100 \%$ of the male patients showed atherosclerotic lesions; whereas $25 \%$ of the female patients had plaques. The prevalence of renal artery calcium was significantly higher in men $(20.2 \%)$ than women $(15 \%)$ in the study of Allison et al. [3]. Tolkin et al. [28] also described a significant relationship between male gender and renal artery calcification. There are also studies in the literature which state that there was no relationship between atherosclerotic plaque presence and gender $[13,15]$.

In our study, $73.6 \%$ of the males and $64.1 \%$ of the females had atherosclerotic plaques and these results were not statistically significant. More plaques were found in the distal part of abdominal aorta, CIAs, EIAs and IIAs in males $(p<0.001)$.

\section{The type of the atherosclerotic plaques}

Tolkin et al. (2009) [28] reported that renal artery calcifications were mild in $58 \%$, moderate in $14 \%$ and severe in $5 \%$ of the patients. In the literature, no other studies were found about the prevalence of the type of the atherosclerotic plaques in abdominal aorta. In our study, $42.9 \%$ of the atherosclerotic plaques were mixed, $23.75 \%$ of them were calcified and $2.68 \%$ of them were soft plaques. The most common type was mixed type and the least common type was soft type and these results were statistically significant $(p<0.001)$.

\section{The burden of the atherosclerotic plaques}

The occlusive lesions were commonly found at the main trunks. Reiner et al. (cited in Dick et al., 1967 [9]) found that 46 of the 88 patients had severe atherosclerosis in aorta, 15 of them had the severest grade of mesenteric artery disease and 10 of them had little or no mesenteric artery disease $[9,12]$. Thomas et al. [27] examined 980 abdominal aortograms and categorised the stenoses of the CT, SMA and IMA in three groups: mild (1-49\%), moderate (50-74\%) and severe (75-99\%) stenoses. They studied the moderate and the severe groups and found that stenosis occurred more frequently in CT (96\%) than in the SMA (50\%) and IMA (57\%) [27]. Valentine et al. [29] used the same method for the classification of the degree of arterial stenosis; $27 \%$ of the 205 male patients had at least one severe mesenteric artery stenosis, $25 \%$ had severe stenosis in CT, $6 \%$ had severe stenosis in SMA and $24 \%$ had moderate and $40 \%$ of them had severe renal artery stenosis.

The recent studies determined the degree of the stenosis instead of classifying the burden of the plaques. In our study, the severities of the plaques were evaluated in a different way. The sizes of the plaques were measured and they were categorised according to their proportions, taking the size of the vessels into consideration. All of the arteries except for IMA mostly had mild plaques; second common type was medium plaques and the least common type was severe plaques for these arteries except for the distal part of abdominal aorta, SMA and IMA. In the distal part of abdominal aorta, severe and at least medium plaques were seen $(p<0.001)$. The burden of the atherosclerotic plaques did not increase with aging.

\section{The atherosclerotic plaques in the branching points}

Atherosclerotic plaques are more frequent around the arterial bifurcations than in the nonbranched portions of the arteries $[6,7,9,26]$. Renal artery calcium was found more common at the renal ostia (15.6\% and $79 \%$ in the studies of Allison et al. [3] and Tolkin et al. [28], respectively) than that in the other parts of the artery. The common outcome of almost all of the studies about atherosclerosis signified that the plaques were most frequently found in the branching points of abdominal aorta. In our study, 109 of $261(41.8 \%)$ patients had plaques in the branching points. The presence of the plaques increased with aging, because of the inadequate number of patients over 81 years, the percentage in this group was low (1.5\%). The atherosclerotic plaques were most commonly found in the aortic bifurcation (73.4\%), secondly in the aortic orifice of CT $(45.9 \%)$ and least commonly in the aortic orifice of IMA (8.3\%) and these differences were statistically significant. 
The co-occurrence of the atherosclerotic plaques in the abdominal aorta and the main branches

DeBakey et al. [6, 7] defined a fifth category for the combination of two or more categories in the $5.7 \%$ of the patients. Atherosclerotic plaques in the branches may extend to aortic plaques but the contrary situation didn't occur $[9,12]$. Calcified atherosclerosis of renal arteries was highly associated with atherosclerosis of aorta [3, 28]. In our study, atherosclerosis of abdominal aorta (proximal, middle or distal) accompanied by the atherosclerosis in the mesenteric vessels or renal arteries but contrary situation had not been observed. Because the number of patients with plaques in the CT and IMA was inadequate, the results were lower than expected.

Atherosclerosis is the main cause of CT, SMA and IMA stenosis and the stenosis of these branches may lead to acute or chronic mesenteric ischaemia. Visceral arterial thrombosis in the atherosclerotic regions is the most common reason for acute mesenteric ischaemia. The stenosis of SMA typically occurs in its proximal part of the artery and the most commonly affected part of the gastrointestinal system is the iliocoecal region [22]. Acute stenosis of SMA is usually fatal but chronic stenosis or obstruction may be compensated by the collateral network between these arteries. The decline in the splanchnic blood flow characteristically causes intestinal angina $[4,19$, 30]. Atherosclerosis of the SA is one of the aetiological factors of splenic infarction and it may cause blood accumulation in the spleen and splenomegaly [5].

The infrarenal part of abdominal aorta is prone to atherosclerosis and aneurysm. Compensatory expansion and conservation of the lumen diameter may accompany plaque formation; if it doesn't, the erosion of the lamellar organisation of tunica media and plaque formation may result in aneurysm [14, 31]. The incidence of myocardial infarction and diabetes mellitus may increase the severity of the mesenteric atherosclerosis. Also hypertension is related with mesenteric atherosclerosis $[9,12]$.

In our study, the prevalence, localisation, type and severity of the atherosclerotic plaques in the parts and branches of abdominal aorta and the aortic orifices and also their association with age and gender were evaluated in a far more comprehensive way than the recent studies. This study also considered the whole population regardless of age, gender, ethnicity or any disease. Knowing the localisation of the atherosclerotic plaques in abdominal aorta (the preferred branches and parts) is extremely important to predict the clinical results and choose the proper treatment (conservative, medical or surgical) or preventing the progress of the disease and the clinical syndromes as mesenteric artery stenosis, renal artery stenosis and splenic infarction. The type and the severity of the plaques also affect the degree of stenosis and the treatment options. A different path was followed for evaluating the severity of the plaques; recent studies had categorised the degree of the stenosis, whereas in our study the plaques were categorised as mild, medium and severe according to the size and extensiveness of them. Our study is the first in the literature in that respect. Also this is the only study in the literature about the prevalence of the types of the atherosclerotic plaques in abdominal aorta. The results of our study is illuminative for evaluating the treatment options in general population, besides that this study will shed light on the future studies about this subject. For the high percentages which are not statistically significant, the number of patients should be increased.

\section{CONCLUSIONS}

In our study, the difference between the proximal part and the other parts of abdominal aorta with respect to plaque existence was statistically significant $(p=0.018)$. More atherosclerotic plaques were found in CIAs than EIAs and IIAs ( $p<0.001$ ), in the LIIA than the LEIA and also in the distal part of the EIA than the other parts.

Proximal parts of the renal arteries had more plaques than the other parts $(p<0.001)$. In the proximal part of splenic artery, less plaques were found $(p<0.001)$. The percentage of the presence of the atherosclerotic plaques increased with age ( $p$ $<0.001$ ). In our study, $64.1 \%$ of females and $73.6 \%$ of males had atherosclerotic plaques; there was no statistically significant difference between genders. More plaques were found in males in the distal part of abdominal aorta and throughout RCIA, LCIA, REIA, LEIA, RIIA and LIIA.

The patients with atherosclerotic plaques most commonly had mixed type (69.3\%), followed by calcified type (42.9\%) and the least commonly had soft type $(23.75 \%$; $p<0.001)$. Most commonly mixed 
plaques were found throughout the abdominal aorta. However, in the CT, SMA, IMA, SA, RRA, LRA, RCIA, LCIA, REIA, LEIA, RIIA and LIIA calcified plaques were the most common type of atherosclerotic plaques.

In the proximal and middle parts of the abdominal aorta and in all of the branches that were included in the study, except for the IMA, mild plaques were the most common, medium plaques were the second common and severe plaques were the least common type. For the distal part of abdominal aorta, the most common type was mixed, second common type was severe and the least common type was medium plaques $(p<0.001)$. The burden of the atherosclerotic plaques did not increase with age.

The atherosclerotic plaques in the branching points were found in the $41.8 \%$ of the patients. Plaque presence in the branching points increased with age. According to the results, $43.8 \%$ of the male patients and $39.3 \%$ of the female patients had plaques in the branching points. There was not any statistically significant difference between genders. The plaques were most commonly found at the aortic bifurcation, secondly at the $\mathrm{CT}$ and least commonly at the IMA $(p<0.001)$. Atherosclerosis of abdominal aorta (any part of it) accompanied by the atherosclerosis in the mesenteric vessels or renal arteries but contrary situation had not been observed.

\section{Acknowledgements}

This study was presented in the 11th Congress of European Association of Clinical Anatomy, Padova, Italia as an oral presentation.

\section{REFERENCES}

1. Aggarwal A, Kapoor K, Singh B (2009) Prevalence and severity of atherosclerosis in renal artery in Northwest Indian population: an autopsy study. Surg Radiol Anat, 31: 349-356. doi:10.1007/s00276-008-0452-0.

2. Allison MA, Budoff MJ, Nasir K, Wong ND, Detrano R, Kronmal R, Takasu J, Criqui MH (2009) Ethnic-specific risks for atherosclerotic calcification of the thoracic and abdominal aorta (from the Multi-Ethnic Study of Atherosclerosis). Am J Cardiol, 104: 812-817. doi:10.1016/j. amjcard.2009.05.004.

3. Allison MA, DiTomasso D, Criqui MH, Langer RD, Wright CM (2006) Renal artery calcium: relationship to systemic calcified atherosclerosis. Vasc Med, 11: 232-238. doi: $10.1177 / 1358863 \times 06073449$.

4. Cunningham CG, Reilly LM, Rapp JH, Schneider PA, Stoney RJ (1991) Chronic visceral ischemia three decades of progress. Ann Surg, 214: 276-287.

5. De Schepper AM, Vanhoenacker F, de Beeck BO, Gielen J, Parizel P (2005) Vascular pathology of the spleen, part II.
Abdominal Imaging, 30: 228-238. doi: 10.1007/s00261004-0267-3.

6. DeBakey M, Glaeser D (2000) Patterns of atherosclerosis: effect of risk factors on recurrence and survival analysis of 11,890 cases with more than 25-year follow-up. Am J Cardiol, 85: 1045-1053.

7. DeBakey MM, Lawrie GM, Glaeser DH (1985) Patterns of atherosclerosis and their surgical significance. Ann Surg, 201: 115-131.

8. Derrick J, Pollard H, Moore R (1959) The pattern of arteriosclerotic narrowing of the celiac and superior mesenteric arteries. Ann Surg, 149: 684-689.

9. Dick Ap, Graff R, Gregg DM, Peters N, Sarner M (1967) An arteriographic study of mesenteric arterial disease I Large vessel changes A. Gut, 8: 206-220.

10. Ditomasso D, Carnethon MR, Wright CM, Allison MA (2010) The associations between visceral fat and calcified atherosclerosis are stronger in women than men. Atherosclerosis, 208: 531-536. doi: 10.1016/j.atherosclerosis.2009.08.015.

11. Frangos S, Gahtan V, Sumpio B (1999) Localization of atherosclerosis role of hemodynamics. Arch Surg, 134: 1142-1149.

12. Glagovt S, Ozoaf A (1968) Significance of the relatively low incidence of atherosclerosis in the pulmonary, renal, and mesenteric arteries. Ann NY Acad Sci, 149: 940-955.

13. Hansen KJ, Wilson DB, Craven TE, Pearce JD, English WP, Edwards MS, Ayerdi J, Burke GL (2004) Mesenteric artery disease in the elderly. J Vasc Surg, 40: 45-52. doi: 10.1016/j.jvs.2004.03.022.

14. Hayashi K, Mani V, Nemade A, Aguiar S, Postley JE, Fuster V, Fayad ZA (2010) Variations in atherosclerosis and remodeling patterns in aorta and carotids. J Cardiovasc Magnet Reson, 12: 10. doi: 10.1186/1532-429X-12-10.

15. Honkanen E, Kauppila L, Wikstrom B, Rensma PL, Krzesinski JM, Aasarod K, Verbeke F, Jensen PB, Mattelaer $P$, Volck B, Group Cs (2008) Abdominal aortic calcification in dialysis patients: results of the CORD study. Nephrol Dial Transplant, 23: 4009-4015. doi: 10.1093/ndt/gfn403.

16. Horton K, Fishman E (2002) Volume-rendered 3D ct of the mesenteric vasculature: normal anatomy, anatomic variants, and pathologic conditions. RadioGraphics, 22: 161-172.

17. Horton KM, Fishman EK (2007) Multidetector CT angiography in the diagnosis of mesenteric ischemia. Radiol Clin North Am, 45: 275-288. doi: 10.1016/j.rcl.2007.03.010.

18. Jairvinen O, Laurikka J, Sisto T, Salenius J, Tarkka MR, Lindholm T (1996) Atherosclerosis in the abdominal aorta and its visceral branches: associations with other manifestations of atherosclerosis in an autopsy study. Int J Angiol, 5: 41-44.

19. Levy AD (2007) Mesenteric ischemia. Radiol Clin North Am, 45: 593-599. doi: 10.1016/j.rcl.2007.04.012.

20. Libby $P$ (2008) The pathogenesis, prevention and treatment of atherosclerosis. In: Fauci AS, Braunwald E, Kasper DL, Hauser S, Longo DL, Jameson JL ed. Harrison's Principles of Internal Medicine. 17 Ed. McGraw Hill, New York, pp. 1501-1509.

21. Malcom G, Oalmann M, Strong JP (1997) Risk factors for atherosclerosis in young subjects: The PDAY Study. Ann NY Acad Sci, 817: 179-188. 
22. Marston A (1964) Patterns of intestinal ischaemia. Ann $R$ Coll Surg Eng, 35: 151-181.

23. Oyama N, Gona P, Salton CJ, Chuang ML, Jhaveri RR, Blease SJ, Manning AR, Lahiri M, Botnar RM, Levy D, Larson MG, O'Donnell CJ, Manning WJ (2008) Differential impact of age, sex, and hypertension on aortic atherosclerosis: the Framingham Heart Study. Arterioscler Thromb Vasc Biol, 28: 155-159. doi:10.1161/ATVBAHA.107.153544.

24. Roberts Jj, Moses C, Wilkins R (1959) Autopsy studies in atherosclerosis: I. Distribution and severity of atherosclerosis in patients dying without morphologic evidence of atherosclerotic catastrophe. Circulation, 20: 511-519.

25. Roberts Jj, Wilkins Rh, Moses C (1959) Autopsy studies in atherosclerosis: II. Distribution and severity of atherosclerosis in patients dying with morphologic evidence of atherosclerotic catastrophe. Circulation, 20: 520-526.

26. Smedby O (1996) Geometric Risk factors for atherosclerosis in the aortic bifurcation: a digitized angiography study. Ann Biomed Engineering, 24: 481-488.
27. Thomas JH, Blake K, Pierce GE, Hermreck AS, Seigel E (1998) The clinical course of asymptomatic mesenteric arterial stenosis. J Vasc Surg, 27: 840-844.

28. Tolkin L, Bursztyn M, Ben-Dov IZ, Simanovsky N, Hiller N (2009) Incidental renal artery calcifications: a study of 350 consecutive abdominal computed tomography scans. Nephrol Dial Transplant, 24: 2170-2175. doi: 10.1093/ $\mathrm{ndt} / \mathrm{gfp} 051$.

29. Valentine RJ, Martin JD, Myers SI, Rossi MB, Clagett GP (1991) Asymptomatic celiac and superior mesenteric artery stenoses are more prevalent among patients with unsuspected renal artery stenoses. J Vasc Surg, 14: 195-199. doi: 10.1067/mva.1991.29423.

30. Van Bockel JH (2001) Chronic splanchnic ischaemia. Best Practice Res Clin Gastroenterol, 15: 99-119.

31. Xu CS, Zarins CK, Glagov S (2001) Aneurysmal and occlusive atherosclerosis of the human abdominal aorta. J Vasc Surg, 33: 91-96. doi: 10.1067/mva.2001.109744. 